\title{
Agronegocios
}

Nota técnica

\section{Subasta ganadera evaluada como proyecto de inversión propuesta por la Cámara de Ganaderos de Upala}

Livestock auction evaluated as an investment project by the

Upala Chamber

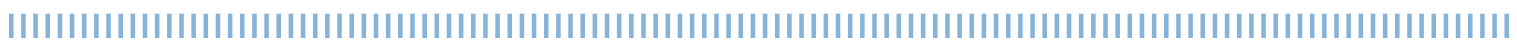

Lucía Morales Abarca ${ }^{1}$

Diana Álvarez Varela

Ignacio Sánchez Gómez 3

Rubén Almendarez Midence ${ }^{4}$

Siany Guillén Castro 5

Fecha de recepción: 05 de agosto, 2019 Fecha de aprobación: 29 de octubre, 2019

Vol.6 No 1 Enero- julio 2020

Almendarez, R; Álvarez, D; Guillén,S; Morales, L; Sánchez, I. (2020). Subasta ganadera evaluada como proyecto de inversión propuesta por la Cámara de Ganaderos de Upala. Revista E-Agronegocios, 6(1). https: https://revistas.tec.ac.cr/ index.php/eagronegocios/article/view/4940

DOI: https://doi.org/10.18845/rea.v6i1.4940

Universidad de Costa Rica. Costa Rica. Bachiller en Economía Agrícola y Agronegocios.

correo electrónico: analu.moab@gmail.com

Universidad de Costa Rica. Costa Rica. Bachiller en Economía Agrícola y Agronegocios

correo electrónico: diana.alvarezvarela@ucr.ac.cr

3 Universidad de Costa Rica. Costa Rica. Bachiller en Economía Agrícola y Agronegocios

Correo electrónico: jose.sanchezgomez@ucr.ac.cr

4 Universidad de Costa Rica. Costa Rica. Bachiller en Economia Agricola y Agronegocios.

Correo electrónico: almendradez_88@hotmail.com 


\section{Resumen}

La Cámara de Ganaderos de Upala es una organización conformada por 140 productores debidamente registrados, con dos años de fundación. Creada con el fin de solidarizarse con los productores después del huracán Otto, ocurrido en 2016 afectando la zona Note de Costa Rica. El proyecto que se plantea es un análisis económico y técnico para el posible establecimiento de una subasta ganadera en la zona de Upala, Costa Rica para el año 2019. El elemento diferenciador sería un margen de comisión competitivo ante las subastas cercanas y una administración directa que beneficia a la cámara y a los ganaderos oferentes de ganado. Se realizó mediante revisión de información secundaria, consulta a expertos, visitas programadas y generación de indicadores financieros, esto con el fin de generar un marco contextual de los alcances de la actividad desde un ángulo financiero y respecto a los principales requerimientos técnicos para una operación máxima de la actividad en estudio. Los principales resultados generados de la investigación fueron: una inversión inicial de c13 717 644,65; donde no se incurre en desembolsos de infraestructura y otros rubros que aumentan esta partida, una tasa interna de retorno positiva de $32,92 \%$, un valor actual neto de c2 615 472,31 derivado de la actividad mediante una tasa de costo de capital de $15,01 \%$ y una un período de retorno de 3.45 años. Como recomendación, la implementación de este proyecto analizado desde los enfoques técnicos y económicos muestra que existe viabilidad para realizar el proyecto, el cual debido a su magnitud es una opción que potencia el fortalecimiento de la cámara y les brinda preparación para proyectos futuros de mayor tamaño.

\section{Abstract}

The Chamber of Cattlemen of Upala is an organization formed by 140 producers, with two years of foundation. Created in order to show solidarity with producers after Hurricane Otto, which occurred in 2016 affecting the north area of Costa Rica. The project is an economic and technical analysis for the possible establishment of a livestock auction in the area of Upala, Costa Rica for the year 2019. The differentiating element would be a competitive commission margin before the nearby auctions and a direct administration that it benefits the camera and the cattle ranchers.

The project was carried out by reviewing secondary information, expert consults, scheduled visits and financial indicators, this in order to generate a contextual framework of the scope of the activity from a financial angle and with respect to the main technical requirements for maximum operation.

The main results of the investigation were: an initial investment of c 13717 644,65; where infrastructure disbursements and other items that increase this item are not incurred, an internal positive rate of return of $32,92 \%$, a value current net of \& 2615472,31 derived from the activity through a capital cost rate of $15,01 \%$ and a return period of 3.45 years.

As a recommendation, the implementation of this project analyzed from the technical and economic approaches shows that there is feasibility to carry out the project, which due to its magnitude is an option that enhances the strengthening of the chamber and provides them with preparation for future larger projects.

Key words: cattle auction, technical analysis, financial analysis, livestock, financial indicators.

Palabras clave: subasta ganadera, análisis técnico, análisis financiero, ganadería, indicadores financieros. 


\section{Introducción}

El presente artículo muestra una serie de análisis sobre los requerimientos técnicos y financieros para el establecimiento de una subasta ganadera en el cantón de Upala, considerando todas las inversiones necesarias en equipo y mano de obra, así como los comportamientos históricos de los precios y los escenarios que podrian afectar la actividad reflejando indicadores financieros que permiten la toma de decisión para llevar a cabo el proyecto.

Inicialmente, el territorio de Upala se ubica en la zona norte del país, es el cantón número 13 de la provincia de Alajuela, creada bajo el decreto de ley N4541 del 17 de marzo del año 1970. Este cantón cuenta con 1580,67 Km2 y una población de 53371 habitantes, proyectada al 2019 (INEC, 2011).

Es importante destacar que el proyecto fue evaluado en la zona de central de Upala, el cuál es un territorio en que prevalecen dentro de las principales actividades productivas la cría, el desarrollo y la comercialización de ganado en pie, principalmente destinado al mercado de cárnicos para consumo nacional, representando este aproximadamente un 5\% de la producción nacional (CORFOGA, 2019).

Por esta razón, es común encontrar en los alrededores de la zona, diversas fincas con infraestructura para el manejo del ganado, sin embargo, muchas de ellas son rústicas y no cumplen con los requerimientos técnicos y legales para el establecimiento de un negocio tipo subasta, no obstante, se da el caso en el que uno de los socios de la Cámara de Ganaderos de Upala cuenta con instalaciones que cumplen con la mayoría de los requerimientos especificados en la normativa $N^{\circ} 34976$ MAG-MEIC-SP (Sistema de Información Jurídica, 2019), que se refiere a los requisitos para el funcionamiento de las subastas a nivel nacional.

Considerando el caso especifico de la Cámara de Ganaderos de Upala y su reciente conformación oficial, es que se evalúa el negocio de una subasta ganadera debido a la creciente necesidad de brindar una respuesta a los asociados frente a la presente inconformidad sobre los negocios similares de la zona. Se desarrolla la opción de la subasta debido a su bajo requerimiento de financiamiento y riesgo; condiciones que se consideran un buen inicio como proyecto de inversión para fortalecer la integridad de la Cámara y como fuente generadora de ingresos en el corto plazo, mientras se consolida la organización y se obtiene experiencia para el embarque a nuevos proyectos de mayor escala como lo son el establecimiento de mataderos y salas de deshuese, que son potenciales actividades para verticalizar la cadena de la actividad ganadera. 


\section{Metodología}

Se realizaron entrevistas a miembros de la Corporación Ganadera (CORFOGA) en donde se obtuvieron principalmente datos referentes a precio y estado del hato nacional. Además, se complementó con consulta a expertos relacionados con la actividad como la actual administradora de la Subasta Ganadera de Puriscal (UPAP), colaboradores de la Asociación de Ganaderos del Distrito Central de Turrialba (ASOTURGA), el economista agrícola, Byron Espinoza colaborador de la Asociación Cámara de Ganaderos Independientes de la Zona Sur y el zootecnista, Diego Cordero de la Universidad de Costa Rica. En la consulta de los indicadores financieros, se contó con el apoyo de la MBA. Olga Maria Calvo Hernández, experta en finanzas de la Universidad de Costa Rica. Finalmente, se realizó en conjunto, los investigadores y la Cámara de Ganaderos de Upala, una visita de campo a la zona potencial donde puede operar la actividad para realizar un diagnóstico de las caracteristicas del inmueble.

Como herramientas para el análisis financiero se utilizaron los indicadores descritos a continuación:

Tasa interna de Retorno (TIR): este indicador evalúa el rendimiento del proyecto en función de una tasa de rendimiento única para todo el horizonte de evaluación en valor presente, la tasa de interés más alta que el inversionista podría pagar sin perdidas económicas. El criterio de evaluación es que, si la TIR es mayor o igual que la tasa de costo de capital, el proyecto resulta rentable para el inversionista y este aprueba el desembolso inicial (Sapag \& Sapag, 2008). (Ecuación 1).

$$
\Sigma=\mathrm{B}_{\mathrm{t}}-\mathrm{C}_{\mathrm{t}} /(1+\mathrm{r})^{\mathrm{t}}=\mathrm{O}
$$

Valor Actual Neto (VAN): este indicador muestra el beneficio neto de la actividad al final del horizonte de evaluación, en donde el criterio de evaluación es que si el VAN es igual o superior a cero el proyecto puede aceptarse sin traducirse esto en pérdidas para el inversionista (Sapag \& Sapag, 2008). (Ecuación 2).

$$
\Sigma=\mathrm{B}_{\mathrm{t}}-\mathrm{C}_{\mathrm{t}} /(1+\mathrm{r})^{\mathrm{t}}-\mathrm{I}_{\mathrm{o}}
$$

Tasa de Costo de Capital: El costo de capital corresponde a aquella tasa que se utiliza para determinar el valor actual de los flujos futuros que genera un proyecto y representa la rentabilidad que se le debe exigir a la inversión por renunciar a un uso alternativo de los recursos en proyectos de riesgos similares.(Sapag \& Sapag, 2008) (Ecuación 3). Se utilizan para poder calcular el costo de caf pital indicadores como lo es el beta apalancado. (Ecuación 4). Posteriormente al cálculo, se debe re convertir la tasa de costo de capital de la moneda extranjera a la nacional, esto mediante la fórmula correspondiente. (Ecuación 5).

$$
\begin{gathered}
K e=R f+\beta^{*}(K m-R f)+R \\
\beta_{a p}=\left(1+(1-T)^{*} D / E\right)^{*} \beta_{d s p} \\
K e_{c}=\left[(1+K e)^{*}\left(1+\text { Inflación }{ }_{C R}\right)\right] / \text { Inflación } u s A
\end{gathered}
$$


Periodo de Recuperación de la Inversión (PRI modificado): este indicador permite medir el plazo en el que se puede recuperar la inversión inicial dentro del flujo del efectivo de un proyecto, teniendo en cuenta el valor del dinero en el tiempo (Solé, 2011).

\section{Resultados y discusión Estimación de Oferta y Demanda}

Al evaluar un negocio de tipo subasta se debe considerar inicialmente, que se parte del hecho de que la oferta y la demanda son consideradas como una igualdad, esto debido a que el tránsito de los animales durante la actividad no implica su estancia en los corrales, ya que una vez que la sesión de puja culmina, si no existiese una oferta por el mismo debe ser retirado de los corrales por el oferente por lo que al cierre de la sesión, los corrales deben estar totalmente vacíos.

A esto existe una excepción, cuando la organización define que dentro de sus funciones como administradora de la actividad, está el hacerse cargo del mantenimiento de los semovientes no vendidos, situación que, para la Cámara de Ganaderos de Upala, no es posible, debido a que la infraestructura en donde se planea desarrollar la actividad no es propia y se planea el alquiler de su uso de manera mensual. Esto es parte del inicio escalonado que se prevé para la subasta ganadera, una actividad con una afluencia baja de unidades animales, especialmente por las condiciones de sequía que afectan la zona en época seca, aunado al incremento en los costos que implicaría el mantenimiento de las reses.

Se obtuvo que para el área de Upala se parte de un valor de 59454 animales en la zona, de los cuales, según consulta a expertos de la Asociación de Ganaderos del Distrito Central de Turrialba (ASOTURGA), un 15\% de este valor es el esperado para el año 1 de la subasta, esto representa un total de 8918 animales, de los cuáles, un 35\% representa a la cantidad de machos en la zona y un $65 \%$ son las hembras.

Al considerar que no todos los animales son homogéneos en cuanto a edad se refiere, se parte de la distribución por edad traslapada del total de animales de Costa Rica a la zona específica de Upala, por lo tanto, en el cuadro número 1 se muestra la distribución de frecuencia de acuerdo a la madurez del animal.

Cuadro 1. Peso promedio por edad del ganado y valores esperados en la zona de Upala, Costa Rica.

\begin{tabular}{lcccc}
\hline Edad & $\begin{array}{c}\text { Peso aproximado } \\
\text { (Kilogramo) }\end{array}$ & $\begin{array}{c}\text { Porcentaje de } \\
\text { animales por } \\
\text { peso en Upala }\end{array}$ & $\begin{array}{c}\text { N de } \\
\text { animales } \\
\text { totales }\end{array}$ & $\begin{array}{c}\mathbf{1 5 \%} \\
\text { esperado }\end{array}$ \\
\hline Menos de un & 150 & $24 \%$ & 14358 & 2154 \\
año & 250 & $27 \%$ & 16037 & 2405 \\
Entre 1 y 2 años & 350 & $49 \%$ & 28911 & 4337 \\
Más de 2 años & & &
\end{tabular}

Con respecto al precio de subasta por kilogramo en pie, este se obtuvo, como un valor promedio de los precios históricos, los datos son mostrados en la figura 1. Para esto, se toman las cifras promedio del último lustro, encontrándose un valor de kilogramo en pie para hembras de c927 y "1104 por kilogramo en pie para machos. 
Figura 1. Tendencia y precios promedios anuales de ganado en colones por kilogramo en pie, según el sexo durante el período 2007 - 2019.

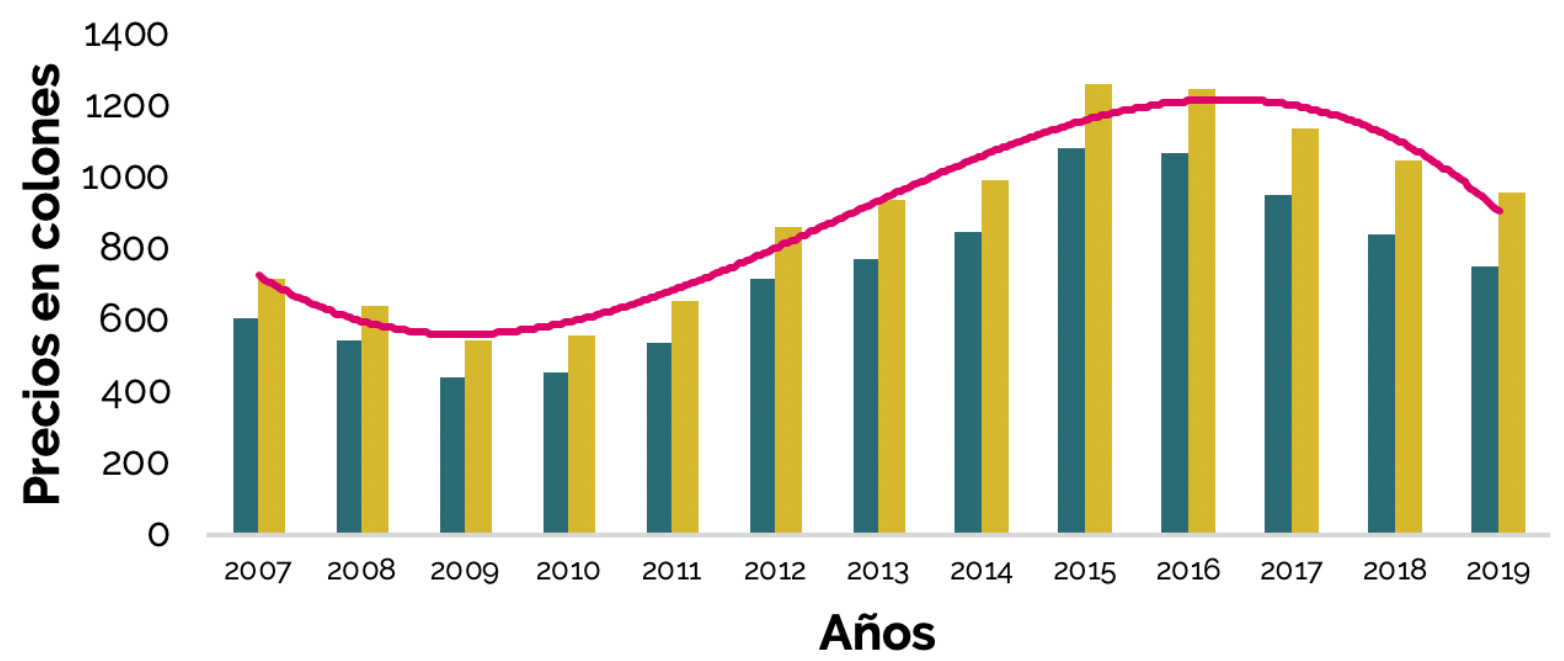

\section{nembras macho}

\section{Análisis técnico}

El proyecto pretende realizar la actividad una vez por semana, donde la puja de los animales sería programada con cada productor con el fin de mantener el orden, no sobrecargar la capacidad de las instalaciones y evitar el estrés en los animales. Cada animal que entra a las instalaciones es revisado por un corralero para posteriormente, ser evaluado por el regente veterinario, quién evalúa la salud y condición del mismo. Una vez aprobado el vacuno, se pasa a los corrales donde debe esperar su turno para ser subastado, en este intervalo de tiempo, serán atendidos por más corraleros quienes les brindarán las condiciones óptimas evitando el estrés y pérdida de peso. Una vez que pase a la mesa de exhibición, un personero de la subasta se encargará de fijar el precio base y ahi empezará la puja hacia el alza hasta ser vendido. El comprador debe pagar por su nuevo bien en las oficinas administrativas para posteriormente dar salida al rumiante.

La infraestructura a utilizar es propiedad privada, la cual está a disposición de alquiler. Se realizó una evaluación del lugar y entre las falencias de la infraestructura se pudo identificar: la falta de áreas acondicionadas para el establecimiento de oficinas, además se debe adecuar una cabina para que el subastador desempeñe su función, se debe adecuar el piso de uno de los apartos con cemento para poder ubicar al público de la subasta, con el fin de que este pueda observar la pantalla que proyecta los precios y la mesa de exhibición, formando parte de la inversión necesaria para la puesta en marcha del proyecto.

La proyección de la inversión inicial del proyecto es de c13 717 586,65. Los activos fijos que forman parte de la inversión inicial, se dividen principalmente en mobiliario para las oficinas, equipo de audiovisual, equipo de pesaje y otros activos menores como lo son palas y empujadores eléctricos.

Los costos en los que incurre el proyecto, requieren: mano de obra, gastos operativos administrativos y gastos operativos en las ventas. El capital de trabajo se calculó en base a los costos 
a los costos desembolsables por período de desfase, que se estableció en una semana debido a periodicidad de la actividad.

El objetivo principal que busca alcanzar la Cámara de Ganaderos de Upala es reducir el porcentaje de comisión como intermediario en la comercialización de los animales en pie, con el fin de mejorar las condiciones para los productores de la zona y comenzar un proceso de reactivación del gremio ganadero en la zona, ya que en los últimos años, la práctica ha tenido un comportamiento decreciente. Actualmente, la mayoría de subastas privadas establecen una comisión entre el $4 \%$ y 4.5\%, sin embargo, debido a la naturaleza sin fines de lucro de la Cámara, se logró establecer una comisión más baja, del 3\% sobre el precio fijado en la subasta. Es en base a esta comisión como se calculan los ingresos de la subasta y se comparan con la inversión inicial y costos totales.

Cuadro 2. Datos en términos reales de Inversiones, Costos Totales e Ingresos para el año 1 del proyecto

\begin{tabular}{lc}
\hline Rubro & Monto en colones \\
\hline Inversión inicial & 13717644,65 \\
Costos totales & 68938521,69 \\
Ingresos & 72713600,44 \\
\hline
\end{tabular}

La ubicación de la infraestructura de la subasta es conveniente, ya que se encuentra muy cerca del centro de Upala, el terreno y alrededores son planos con capacidad para extender los corrales si en un futuro es necesario. No es una zona residencial, por lo que no habría problemas con vecinos y adicionalmente, cuenta con todos los servicios básicos (agua, electricidad, teléfono e internet) y la cercanía al centro poblacional le da acceso a diversidad de otros servicios (bancos, clínica, comercio, entre otros). El acceso vial se encuentra en buenas condiciones ya el terreno en cuestión se encuentra al frente de la carretera principal, misma que está asfaltada.

Los factores ambientales también deben ser considerados para el establecimiento del proyecto. Mediante el criterio experto se determinó que, para el manejo de excretas y residuos líquidos de los animales, se colocarán camas de aserrin para posteriormente, ser utilizado como material orgánico en el cultivo de pastos de los mismos asociados en la zona. Esta estrategia fue elegida debido, principalmente, al bajo costo de implementación que requiere. Por otro lado, al alquilar las instalaciones, si se considera en el futuro, necesario un cambio de infraestructura a otro terreno o a un terreno propio, no se tendría pérdidas de inversión por instalaciones de equipo.

El buen manejo y mantenimiento de las instalaciones ayuda a reducir el nivel de estrés de los animales, evitando así la pérdida de peso. Para promover la relajación de las cabezas de ganado, se contará con 3 corrales, en donde se atenderán a 8 animales por hora, de forma que el tránsito sea rápido y eficiente, para evitar ese desgaste o pérdida de peso. Además, se contará con 5 corraleros para la atención y control de estos corrales. Esta cantidad de animales también permite mantener la integridad de los mismos, de forma que no pierdan peso o se vean afectados por combates, ya que estos se dan cuando se mezclan animales de diferentes tamaños y pesos. Las condiciones actuales presentan pasillos donde el ganado se puede movilizar cómodamente, las puertas, portones y mangas se encuentran en condiciones óptimas y las mangas ya se encuentran acondicionadas para su uso. 


\section{Evaluación Financiera \\ Calculo de la Tasa de Costo de Capital}

Dentro de la evaluación financiera de los proyectos el cálculo de la tasa de costo de capital es una de las variables más importantes, ya que permite la estimación del indicador de rentabilidad VAN, uno de los indicadores más comúnmente utilizado en la evaluación de proyectos y de mayor confiablidad cuando la tasa de descuento es calculada de la forma correcta.

Además de lo anterior, la Tasa de Costo de Capital, sirve como parámetro para comparar otro indicador de gran importancia en este campo, como lo es la Tasa Interna de Retorno, así la Tasa de Costo de Capital indica el valor mínimo aceptado por el inversionista como rendimiento del proyecto. En este caso, la Tasa de Costo de Capital es calculada mediante el Modelo Precio Activo de Capital, sin embargo, como el proyecto debe de optar por financiamiento para cubrir su inversión inicial, se ponderaron los costos de las fuentes de financiamiento (pasivo y aporte de capital) y se estableció en un valor de 15,10\%.

\section{Financiamiento}

La Cámara de Ganaderos de Upala se fundó hace poco tiempo (1,5 años aproximadamente), por este motivo no cuenta con el capital propio necesario para cubrir la inversión inicial, que si bien es cierto no es un monto muy elevado, debido principalmente al hecho de no tener que invertir en el levantamiento de la infraestructura ni a la compra de un terreno, no se encuentran en condiciones de asumirlo, por esta razón, se consultó a funcionarios del INDER acerca de los Créditos Rurales que ofrecen facilidades a iniciativas productivas de la zona rural, como lo son una baja tasa de interés fija y plazos acordes a la actividad que se esté desarrollando, tomando en cuenta ciclos productivos.

A continuación, en el cuadro 3, se presentan las condiciones del financiamiento que se establecieron para el análisis, partiendo de un supuesto para la definición del plazo y financiando un $80 \%$ de la inversión inicial.

Cuadro 3. Términos de financiamiento para el 90\% de la inversión inicial

\begin{tabular}{lc}
\hline Condiciones & Valor \\
\hline Préstamo & c10 974 321,32 \\
Tasa de Interés & $5 \%$ \\
Plazo (años) & 5 \\
Cuota Nivelada & ç2 748 589,61 \\
\hline
\end{tabular}

Vale la pena resaltar que, debido a que la Cámara se encuentra exenta del pago del impuesto de renta dentro del flujo de caja no se refleja el efecto del escudo fiscal producido por los ajustes de los gastos no desembolsables como lo son la depreciación de los activos fijos y la amortización de activos de naturaleza nominales.

En el cuadro 4 se muestra el comportamiento del flujo neto de efectivo (FNE) generado a partir de los datos analizados a lo largo del presente documento durante el horizonte de evaluación de 5 años. 


\begin{tabular}{lc}
\hline Condiciones & Valor \\
\hline Préstamo & ¿10 974 321,32 \\
Tasa de Interés & $8 \%$ \\
Plazo (años) & 5 \\
Cuota Nivelada & c2 748 589,61 \\
\hline
\end{tabular}

\section{Indicadores de Rentabilidad}

Para el presente estudio, se utilizarán tres indicadores de rentabilidad: el Valor Actual Neto (VAN), la Tasa Interna de Retorno (TIR) y el Periodo de Retorno de la Inversión ( $\mathrm{PRI} \mathrm{mod}_{\text {od }}$ ) en su variante mejorado. Según Rosales, el VAN es uno de los indicadores más utilizados a la hora de evaluar un proyecto (Rosales, 2017) Por su parte, el mismo autor afirma que la TIR es un elemento de juicio necesario cuando se desea realizar una inversión de forma racional y financieramente eficiente (Rosales, 2017).

Por último, se utilizará el PRI ${ }_{\text {mod' }}$ el cuál es un indicador que identifica el periodo en el que se recupera la inversión inicial, pero a diferencia de su predecesor, este contempla el valor del dinero en el tiempo, por lo que se muestra en el cuadro 5 los valores tomados por los diferentes indicadores.

Cuadro 5. Valores estimados para los indicadores de rentabilidad del proyecto de subasta ganadera en Upala.

\begin{tabular}{lc}
\hline Indicador & Valor \\
\hline Ko & $15,01 \%$ \\
TIR & $29,70 \%$ \\
VAN (10\%) & C2 615 472,31 \\
PRImod & 3,45 \\
\hline
\end{tabular}

Con lo anterior se pudo concluir que después de la estimación del flujo de efectivo, el Valor Actual Neto del proyecto es mayor a cero, lo que quiere decir que el proyecto durante el horizonte de evaluación planteado genera un excedente actualizado para el inversor de c2 615 472,31, por lo cual, basados en que el proyecto no genera pérdidas, debería de aprobarse. Además, se puede concluir según la TIR, que al ser mayor que la Tasa de Costo de Capital el proyecto está generando beneficios económicos para el o los inversores. Por último, el PRI modificado no indica que la inversión inicial se recupera en poco más de 4 años, la decisión en este caso depende de los miembros de la Cámara, ya que ellos deben analizar si esperan este tiempo para recuperar este monto.

\section{Análisis de sensibilidad}

Como método de estimación, se realizó un análisis de sensibilidad bidimensional variando, el porcentaje de participación (kilogramos subastados) en la subasta y el porcentaje de comisión cobrado por parte de la Cámara, obteniendo el cálculo del Valor Actual Neto. En el cuadro 6 se aprecia el efecto de los cambios. 
Cuadro 6. Análisis de sensibilidad bidimensional, variando la comisión cobrada y el porcentaje de participación

\begin{tabular}{|c|c|c|c|c|c|c|}
\hline & & \multicolumn{5}{|c|}{ Porcentaje de participación en la subasta } \\
\hline & & $9 \%$ & $12 \%$ & $15 \%$ & $18 \%$ & $21 \%$ \\
\hline \multirow{5}{*}{$\begin{array}{c}\text { Comisión } \\
\text { por } \\
\text { animal } \\
\text { subastad } \\
0\end{array}$} & $2,00 \%$ & - $\quad 55610714.47$ & - $\quad 70620916,26$ & $-85631118,05$ & $-100641319,85$ & $-115651521,64$ \\
\hline & $2,50 \%$ & - $\quad 29163505,79$ & - $\quad 35357971,35$ & - $\quad 41552436,92$ & - $\quad 47746902,49$ & - 53941368,05 \\
\hline & $3,00 \%$ & 2662760,25 & 23643,97 & 2615472,31 & 5254588,59 & 7893704,87 \\
\hline & $3.50 \%$ & 23784448,43 & 35239300,94 & 46694153,45 & 58149005,95 & 69603858,46 \\
\hline & $4,00 \%$ & 50285193.97 & 70573628,33 & 90862062,68 & 111150497.03 & 131438931,39 \\
\hline
\end{tabular}

El principal resultado de este análisis es la imposibilidad de la Cámara de cobrar una comisión menor al 3\% bajo los parámetros establecidos en este estudio, debido a que un monto inferior a este genera pérdidas económica bajo todos los escenarios de participación, esto es apoyado por las consultas realizadas a expertos durante el desarrollo de este proyecto que indican que las subastas ganaderas privadas cobran, generalmente, alrededor de un $4 \%$ del precio de venta del animal.

Además de lo anterior, se realizó un análisis multidimensional con la herramienta de análisis de datos Solver, para estimar el punto de equilibrio de la actividad bajo los supuestos establecidos en este documento, resultados que se muestran en el cuadro 7.

Cuadro 7. Punto de equilibrio para la subasta ganadera en Upala

\begin{tabular}{lc}
\hline Rubro & Valor \\
\hline Unidades por vender & \\
(Kilogramo/año) & 2451278,68 \\
Precio de venta & \\
(colones/Kilogramo) & 29,38 \\
TIR & $15,10 \%$ \\
VAN (10\%) & - \\
\hline
\end{tabular}

Del siguiente análisis se concluye que, para no producir pérdidas o ganancias, la subasta debe vender 2451 278,68 Kilogramo/año a un precio de equilibrio de c29,38 por Kilogramo, estos valores estiman un monto de VAN de cero y una TIR de 15,10\% la cual es igual que la Tasa de Costo de Capital.

\section{Conclusiones y recomendaciones}

Se logró demostrar que, bajo las condiciones anteriormente expuestas en el análisis de oferta, demanda y la evaluación financiera, este proyecto tiene el potencial de ser una actividad que le genere ingresos a la Cámara y le permita consolidarse como una organización con capacidad de desarrollar proyectos de manera exitosa y que a su vez, fortalezcan la actividad ganadera de la zona norte del país. 
La implementación de la subasta no involucra un financiamiento de gran magnitud ya que se solicita un financiamiento de c13 717 644,65 que puede ser solicitado ante entidades como el Instituto Nacional de Desarrollo Rural (INDER), el Ministerio de Agricultura y Ganadería (MAG), entre otras; todas estas con capacidad de brindar recursos financieros para el desarrollo de actividades rurales. Este proyecto es una buena manera de consolidar la organización y a todos sus miembros al demostrar un buen accionar con la concreción de actividades con un impacto directo e indirecto para el cantón en un corto plazo.

El desarrollo de actividades complementarias al negocio de la subasta como lo son los mataderos y salas de deshuese que tienen la finalidad de verticalizar la actividad ganadera, son proyectos de mayor plazo de realización para los cuales la cámara necesita una articulación inter institucional que les provea los requerimientos técnicos, financieros, de mercado, legal y ambiental para realizar los primeros acercamientos a la evaluación potencial de establecer estos en la zona, para lo cual aún no se cuenta con la madurez organizacional requerida para solicitar financiamientos de dichas magnitudes, las cuales incluyen adquisiciones de terreno e infraestructura, por lo tanto representan un compromiso legal y financiero para el que se debe estar preparado gracias a la experiencia que generan proyectos de menor escala.

Se recomienda desarrollar este proyecto, ya que el aporte de capital puede ser cubierto fácilmente por una contribución individual de cada uno de los miembros de la Cámara, obteniendo de esto un beneficio directo en el ingreso por la actividad al presentarse una reducción de costo por transporte, de pérdida de peso por estrés y finalmente, un reintegro a la sociedad gracias al enriquecimiento de la zona beneficiada la cual se traduce directamente en mayor bienestar en la comunidad. 


\section{Literatura citada}

BCCR. (Junio de 2019). Banco Central de Costa Rica. Obtenido de https://www.bccr.fi.cr/seccion-indicadores-economicos/indicadores-econ\%C3\%B3micos

Fondo para los logros de los ODM. Encuesta sobre la realidad de la persona joven de Upala.2010 recuperado de: https://www.google.com/url?sa=t\&source=web\&rct=j\&url=http://WwW.nacionesunidas.or.cr/dmdocuments/B_Encuesta_Upala.pdf\&ved=2ahUKEwjuubCVn7niAhWN1kKH SWgAGEQFjABegQIAhAB\&usg=AOvVawooDkgBlxLu6YofcLIztTgm

INEC. (2018). Obtenido de http://Www.inec.go.cr/pobreza-y-desigualdad/desigualdad

Instituto de Fomento y Asesoria Municipal (IFAM). Upala. s.f. Recuperado de: http://Www. ifam.go.cr/?page_id $=482$

Instituto Nacional de Estadistica y Censo (INEC)-Costa Rica. 2018. Directorio de Empresas

y Establecimientos. Recuperado de: http://www.inec.go.cr/sites/default/files/infografias-multimedia/imginfo-dee2018.jpg

Instituto Nacional de Estadistica y Censo (INEC)-Costa Rica. s.f.; Indicadores Territoriales. Upala. Recuperado de: http://www.inec.go.cr

Instituto Nacional de Estadistica y Censo (INEC)-Costa Rica.Costa Rica: Población total proyectada al 30 de junio por grupos de edades, según provincia y cantón. 2011-2025 Recuperado de: http://ww/w.inec.go.cr/poblacion/estimaciones-y-proyecciones-de-población

Ministerio de Planificación (MIDEPLAN). 25 de Mayo 2019. Mapa Inversiones Costa Rica.

Upala. Recuperado de: http://mapainversionescr.mideplan.go.cr/localizacion/LocationProfile\#s5

Rosales, R. (2017). La formulación y la evaluación de proyectos con énfasis en el sector agrícola. San Jose: EUNED. Recuperado el Junio de 2019

Sapag, N., \& Sapag, R. (2008). Preparación y evaluación de proyectos. Bogotá, Colombia: McGraw-Hill. Recuperado el Octubre de 2018, de http://www.delfabro.cl/preparacinyevaluacindeproyectos_nassirsapag5edi.pdf

Sistema de información jurídica. (Junio de 2019). sistema de información jurídica. Obtenido de http://www.pgrweb.go.cr/scij/main.aspx

Solé, R. (2011). Técnicas de evaluación de flujos de inversión: mitos y realidades. Revista UCR, 426. Recuperado el Octubre de 2018, de https://revistas.ucr.ac.cr/index.php/economicas/article/ viewFile/7051/6736 